\title{
Uso terapéutico de los cannabinoides
}

\author{
Marta Duran Delmàs'; Dolors Capellà Hereu² \\ 1. Médico adjunto de la Fundación Institut Català de Farmacología. Hospitals Vall d’Hebron. \\ 2. Profesora titular de Farmacología Toxicología y Terapéutica. Universidad Autónoma de Barcelona. \\ Enviar correspondencia a: \\ Marta Duran Delmàs. Fundació Institut Català de Farmacologia. Escola d'infermeria 3er Piso. Servicio de Farmacología Clínica. Hospitals Vall d'Hebron. Pg Vall \\ d'Hebron 119-129. Barcelona 08035. Tel: 93.4283029. Fax: 93. 4894109. e-mail: mdd@icf.uab.es. \\ Recibido: abril 2004. \\ Aceptado: mayo 2004.
}

\section{RESUMEN}

Actualmente hay dos cannabinoides comercializados en algunos países para uso terapéutico. El dronabinol o D9tetrahidrocannabinol (THC) y su análogo babilona para el tratamiento de las náuseas y vómitos secundarios a la quimioterapia antineoplásica que no responde a los tratamientos habituales; pero su lugar en terapéutica como antieméticos ha sido cuestionado porque no se dispone de ensayos clínicos comparativos con los tratamientos de referencia (inhibidores de la serotonina). El dronabinol también está aprobado en algunos países para el síndrome de anorexiacaquexia de pacientes con sida. Aunque la investigación preclínica actual tiende a desarrollar nuevos fármacos que interactúen con el sistema cannabinoide, la investigación clínica se está realizando sobretodo con THC y extractos estandarizados de cannabis (que contienen mayoritariamente THC y cannabidiol). Empiezan a acumularse pruebas clínicas que sugieren que los cannabinoides podrían ser eficaces en el tratamiento sintomático del dolor neuropático, la esclerosis múltiple (EM) y en la mejoría de los tics del síndrome de Gilles de la Tourette y determinadas discinesias, sin embargo, habrá que esperar los resultados de los estudios en curso para llegar a conclusiones mejor fundamentadas. En relación al tratamiento sintomático de la EM, los cannabinoides no han mostrado eficacia antiespástica según $>$ variables objetivas, sin embargo algunos datos informan de que puede mejorar la sensación subjetiva de rigidez, el dolor y la calidad del sueño de estos pacientes a la espera de que nuevos ensayos clínicos lo confirmen.

Palabras clave: sistema cannabinoide, cannabis, cannabinoides, dolor, espasticidad, nauseas, vómitos, eficacia, efectos indeseados, revisión.

\section{ABSTRACT}

At the present time, there are two cannabinoids approved for clinical use in several countries. Dronabinol or D9tetrahidrocannabinol (THC) and its classical synthetic analogue nabilone for antineoplastic chemotherapy-induced secondary nausea and vomiting which does not respond to the usual treatment but their place in therapeutics as an antiemetic has been questioned because there is no available data from clinical trials comparing them with standard treatment (serotonine inhibitors). Dronabinol is also approved in certain countries for anorexia associated with weight loss in AIDS patients. Although current preclinical research is developing new pharmaceuticals that interact with the cannabinoid system, clinical research is concentrating principally on $\mathrm{THC}$ and standardised cannabis extracts (which mainly contain THC and cannabidiol). Clinical trials suggest that the cannabidiols could be effective in the symptomatic treatment of neuropathic pain, multiple sclerosis (MS) and in the tics associated with the Giles de la Tourette syndrome and certain dyskinesias, but the results of studies in progress are necessary to be able to draw better founded conclusions. As for the symptomatic treatment of MS, the cannabinoids have not been shown to be effective against spasticity in accordance with objective variables but certain data points to there being an improvement in the subjective sensation of rigidity, pain and the sleep quality in these patients but further clinical trails must be undertaken to confirm this.

Key words: endocannabinoid system, cannabis, pain, spasticity, nausea, vomiting, efficacy, adverse effects, review. 


\section{INTRODUCCIÓN}

$\mathbf{L}$ a planta del cannabis (Cannabis sativa) ha sido utilizada en terapéutica desde la antigüedad. En los últimos años su uso terapéutico ha sido amplia y extensamente debatido. El interés manifestado por algunas asociaciones de pacientes por el uso de dicha planta y sus componentes activos y derivados (cannabinoides) ha reavivado la polémica. Este uso contrasta con la falta de ensayos clínicos que cumplan los requisitos de las agencias reguladoras para aprobar su uso como medicamento'. La realización de estos estudios es difícil por razones logísticas (es difícil conseguir financiación y también la planta o sus extractos), metodológicas (es difícil el enmascaramiento y controlar la variabilidad inter e intraindividual de los efectos de los cannabinoides así como encontrar variables que midan objetivamente mejorías sintomáticas subjetivas como la náusea, el dolor, la espasticidad o la calidad de vida que refieren los pacientes tratados con cannabinoides) y legales (el cannabis es una droga de abuso ilegal en la mayoría de países). Por otro lado, el recién desvelado sistema cannabinoide formado por receptores específicos y moléculas endógenas que se unen a ellos (endocannabinoides) ha contribuido al renacimiento del interés por las propiedades terapéuticas de los cannabinoides. La investigación actual tiende a desarrollar nuevos fármacos que actúen potenciando o inhibiendo los efectos de los endocannabinoides y fármacos sintéticos agonistas y antagonistas de los receptores cannabinoides. Sin embargo, el futuro inmediato de la investigación clínica tiende a basarse en extractos de cannabis con un contenido conocido y estandarizado de principios activos ${ }^{2,3}$, así como en el desarrollo de nuevas vías de administración como la sublingual, transdérmica, inhalada en aerosol o rectal.

El objetivo de este artículo es revisar los resultados de la investigación clínica disponible sobre el uso terapéutico de los cannabinoides así como aportar información sobre los ensayos clínicos en curso en las diversas indicaciones en las que se están evaluando estos compuestos. El contenido se basa en un Informe técnico realizado por la Fundación Institut Català de Farmacologia a petición del Departament de Sanitat i Seguretat Social de la Generalitat de Catalunya ${ }^{4}$ partes del cual han sido publicadas en otras revistas médicas y boletines ${ }^{1,5}$.

\section{COMPOSICIÓN Y DERIVADOS DEL CANNABIS}

El Cannabis sativa es una variedad del cáñamo cultivado desde hace muchos años por sus propiedades médicas y psicoactivas. También se denomina marihuana la mezcla de las partes de la planta desecada, cuyo contenido en cannabinoides es variable y depende del lugar y de las condiciones de cultivo, de reco- lección y desecación. La planta del cannabis contiene más de 400 sustancias, de las cuales 61 tienen estructura de cannabinoide, similar a la de su principio activo más importante, el $\Delta^{9}$-tetrahidrocannabinol (THC). Hay dos cannabinoides comercializados en diversos países para uso terapéutico; el dronabinol o THC disuelto en aceite de sésamo (Marinol, ${ }^{\oplus}$ Elevat ${ }^{\oplus}$ ) y la nabilona (Nabilone ${ }^{\circledR}$ ), un análogo del THC ${ }^{1}$.

El THC es responsable de la mayoría de las acciones psicoactivas y efectos terapéuticos atribuidos al cannabis, como los efectos antiemético, analgésico, sedante y estimulante del apetito. Otros cannabinoides con más o menos relevancia clínica son el $\Delta^{8}$ tetrahidrocannabinol $\left(\Delta^{8} \mathrm{THC}\right)$, el cannabigerol, el cannabidiol (CBD), el cannabinol (CBN) y el cannabicrómeno (CBC). Entre ellos cabe destacar el CBD, un cannabinoide no psicoactivo que actúa por un mecanismo diferente al del THC y comparte algunos de sus efectos terapéuticos. Ha mostrado efecto neuroprotector in vitro ${ }^{6}$, así como efecto analgésico ${ }^{7}$, antiinflamatorio ${ }^{8}$, immunosupresor ${ }^{9}$, antinauseoso ${ }^{10}$, hipnótico y ansiolítico ${ }^{11}$ en modelos de experimentación animal.

Se ha sugerido que en administración conjunta los diferentes cannabinoides de la planta modularían la acción del THC y potenciarían algunos de sus efectos terapéuticos. Esto podría explicar porqué algunos pacientes prefieren el cannabis a los derivados sintéticos, a pesar de que no disponemos de estudios rigurosos que lo confirmen.

\section{FARMACOLOGÍA BÁSICA}

Se han identificado dos receptores cannabinoides y tres familias de substratos endógenos que se les unen con mayor o menor afinidad produciendo los mismos efectos que el THC en modelos de experimentación animal (antinocicepción, inmovilidad, reducción de la actividad espontánea e hipotermia) 12,13,14. El receptor cannabinoide CB1 se encuentra mayoritariamente en el sistema nervioso, sobre todo en los ganglios basales, el cerebelo y el hipocampo. El receptor CB2 se encuentra sobre todo en las células inmunitarias. No se descarta la posibilidad de que existan otros receptores cannabinoides ${ }^{12}$. En 1992 se descubrió el premier endocannabinoide, la araquidoniletanolamida (anandamida). Tres años más tarde se caracterizó el 2-araquidonil-glicerol (2-ARA-G), un endocannabinoide del grupo de los ésteres del ácido araquidónico ${ }^{13}$. El éter de 2-araquidonil-gliceril o noladina es un tercer tipo de endocannabinoide identificado más recientemente ${ }^{14}$.

Durante la última década se han sintetizado centenares de moléculas agonistas y antagonistas de los receptores cannabinoides. Algunas se han comercializado para uso terapéutico y otras se han reservado para investigación básica, lo que ha permitido caracterizar 
la bioquímica y la fisiología del sistema cannabinoide y su función moduladora del sistema nervioso ${ }^{15}$, la inmunidad $^{16}$, el aparato cardiovascular ${ }^{17}$ y la reproducción ${ }^{18}$. Este sistema es un complejo modulador, particularmente importante en el mantenimiento de la homeostasis del organismo ${ }^{19}$.

\section{EFECTOS FARMACOLÓGICOS DEL CANNABIS}

Los términos con los que se describen los efectos agudos del cannabis sobre el sistema nervioso son ligera euforia, relajación, aumento de la sociabilidad, potenciación de las percepciones sensoriales y del hambre. Otros efectos habituales, no tan deseados o buscados con su consumo, son afectación de la memoria, alteración de la percepción del paso del tiempo y menor rendimiento en diversas trabajos motores, como escribir a mano o otras actividades de la coordinación motora, y taquicardia. Puede producir efectos desagradables: la mitad de los usuarios (con finalidad lúdica) han tenido como mínimo un episodio de ansiedad secundario a su consumo, generalmente a dosis altas $^{20}$.

Estudios recientes han sugerido la existencia de una relación entre el uso crónico de marihuana y depresión, ansiedad, esquizofrenia y otras psicosis y alteraciones de la función cognitiva. 21,22,23,24. No obstante hasta el momento actual, no se ha podido demostrar que las alteraciones de la función cognitiva en fumadores crónicos adultos de cannabis sean irreversibles ${ }^{25 ; 26}$.

La relación entre el cannabis y la psicosis está bien establecida. No obstante, no hay pruebas concluyentes que demuestren que pueda producir esquizofrenia. La teoría más aceptada es que podría precipitar un episodio en personas con antecedentes o exacerbar los síntomas en enfermos con esquizofrenia ${ }^{27}$. La relación entre el uso crónico de cannabis y la depresión ha sido menos estudiada. No obstante, dos estudios recientes apoyan la hipótesis de que el uso crónico de cannabis durante la adolescencia podría ser un factor de riesgo de depresión en el adulto ${ }^{21,22}$.

\section{FARMACOCINÉTICA}

La cantidad de THC absorbida depende de la vía de administración. Por inhalación de humo, la absorción es rápida y los efectos se inician en pocos minutos, y son completos antes de media hora ${ }^{28}$. Por vía oral, la biodisponibilidad del THC es de un 5-10\%, debido a que es destruido parcialmente por el jugo gástrico y a que es sometido a metabolismo hepático de primer paso; los efectos se inician entre $1 / 2$ y 2 h después de su ingestión y duran de 2 a $6 \mathrm{~h}^{28}$. Los niveles plasmáticos de THC que se alcanzan tras la administración por vía oral pueden resultar modificados por la presencia de alimentos, sobre todo lípidos, los cuales aumentarían su biodisponibilidad. Se ha sugerido que la administración en aerosol aseguraría una absorción rápida y completa, y evitaría los efectos perjudiciales del humo. También se ha administrado por vía rectal, sublingual y transdérmica ${ }^{28}$.

El THC se distribuye rápidamente por el organismo, primero a los tejidos más irrigados (cerebro, pulmones, hígado). Después se acumula en el tejido adiposo y se va liberando a la sangre. La semivida de eliminación plasmática (t1/2) del THC puede variar entre 28 a $56 \mathrm{~h}$. Se han identificado alrededor de veinte metabolitos del THC, algunos de ellos activos. El principal es el $11-\mathrm{OH}-\Delta^{9}-\mathrm{THC}$, con una t1/2 de 12 a $36 \mathrm{~h}$. Los metabolitos inactivos son eliminados por la orina y las heces. El THC y algunos metabolitos activos son sometidos a circulación enterohepática, y esto contribuye a alargar la duración de su efecto ${ }^{28}$.

\section{EFICACIA CLÍNICA DE LOS CANNABINOIDES}

La eficacia de los cannabinoides ha sido revisada por diversos organismos, entre los que destacan el Comité Científico de la Cámara de los Lores Británi$\mathrm{ca}^{29,30} \mathrm{y}$ el Institute of Medicine (IOM) norteamericano (1999) .

Las indicaciones mejor evaluadas han sido la profilaxis y el tratamiento de las náuseas y vómitos secundarios a la quimioterapia antineoplásica, el síndrome de anorexia-caquexia en pacientes con sida o con ciertos cánceres en fase terminal, el dolor y la espasticidad muscular ${ }^{1}$. Actualmente también se están evaluando como neuroprotectores $^{31}$ y agentes antitumorales ${ }^{32}$. Su eficacia en las alteraciones del movimiento, glaucoma y asma ha sido menos estudiada.

\section{Profilaxis y tratamiento de las náuseas y vómitos secundarios a la quimioterapia antineoplásica}

La eficacia antiemética de los cannabinoides se basa en los resultados de series de pacientes ${ }^{33,34}$, de dos pequeños ensayos clínicos con marihuana fumada ${ }^{35,36}$, y de una revisión de 30 ensayos clínicos con nabilona y dronabinol ${ }^{37}$.

En la década de los ochenta se publicaron los resultados de diversas series de pacientes oncológicos con náuseas y vómitos secundarios al tratamiento citostático tratados con marihuana fumada cedida por el National Institute of Drug Abuse (NIDA) de Estados Unidos $^{33,34}$. Un 70 a $90 \%$ de los pacientes refirieron mejoría con marihuana fumada y THC por vía oral ${ }^{33}$. En otra serie, Vicengueira et al. encontraron que de los 74 pacientes tratados, 18 (24\%) abandonaron el trata- 
miento. De los 56 restantes un $60 \%$ encontraron que el tratamiento era muy o moderadamente efectivo ${ }^{34}$.

Durante la misma década se publicaron dos ensayos clínicos ${ }^{35,36}$ En uno ellos (cruzado, a doble ciego y controlado con placebo), la marihuana fumada administrada cada 4 h durante 24 h mostró una eficacia antiemètica (medida según la preferencia de los pacientes) superior a la de placebo y similar a la de THC administrado por vía oral en 20 pacientes con náuseas y vómitos secundarios al tratamiento citostático. Los efectos indeseados fueron leves, aunque 7 pacientes presentaron alteraciones de la percepción ${ }^{35}$. En otro ensayo clínico cruzado doble ciego y comparado con placebo, en 15 pacientes resistentes a dronabinol, los cigarrillos de marihuana con un contenido de 17,4 mg de THC inhalado cada 3 o 4 h durante los tres días posteriores a la quimioterapia mostraron una eficacia antiemètica (reducción de la frecuencia y la intensidad de las náuseas y los vómitos y del volumen del vómito) superior a la de placebo. La incidencia de nauseas y vómitos en el grupo que recibió placebo fue de un $72 \%$ y en el grupo que recibió tratamiento activo se correlacionó con los niveles plasmáticos de THC. Un $44 \%$ y un $6 \%$ de los pacientes, presentaron náuseas y vómitos cuando los niveles plasmáticos de THC fueron de $<5 \mathrm{ng} / \mathrm{ml}$ o $>10 \mathrm{ng} / \mathrm{ml}$ respectivamente. Los niveles plasmáticos alcanzados por vía oral fueron más variables que los alcanzados por vía inhalada. No obstante, la mayoría de pacientes incluidos en el estudio eran jóvenes y con experiencia de uso previo de cannabis $^{36}$.

Estos estudios presentan algunas limitaciones metodológicas que dificultan sacar conclusiones. Sin embargo, los resultados sugieren que la marihuana fumada produciría una mejoría subjetiva superior a la de placebo y similar a la de THC por vía oral.

Los resultados de un metanálisis de 30 ensayos clínicos, en un total de 1.366 pacientes muestran que la nabilona y el dronabinol tienen una eficacia superior a la de placebo y a la de otros antieméticos, como la proclorperacina o la metoclopramida, en pacientes que han recibido quimioterapia moderadamente emetóge$\mathrm{na}^{37}$. Sobre la base de estos resultados se ha sugerido que la nabilona y el dronabinol podrían ser eficaces sobre todo en la prevención de las náuseas, pero se desconoce actualmente si tienen alguna ventaja sobre los antagonistas de la serotonina en monoterapia o añadidos al tratamiento de referencia, ya que no se han publicado ensayos clínicos comparativos entre ellos, ni tampoco hemos identificado estudios actualmente en curso.

En la mayoría de los ensayos clínicos incluidos en la revisión de Tramèr et al. se registraron más efectos indeseados en los pacientes que recibieron dronabinol o nabilona que en los de los grupos control, siendo los más frecuentes somnolencia, sequedad de boca, vér- tigo, alteraciones visuales y disforia. A pesar de ello, en los ensayos clínicos cruzados los pacientes manifestaron preferencia por los cannabinoides para ciclos posteriores de quimioterapia. Los autores sugieren que determinados efectos indeseados como la sedación y la euforia, podrían considerarse más bien como potencialmente beneficiosos en el contexto, habitualmente angustioso, del paciente oncológico. Sin embargo, otros efectos indeseados más molestos, como el vértigo, la disforia, la depresión, las alucinaciones, la paranoia y la hipotensión, también fueron más frecuentes en el grupo tratado con cannabinoides, aunque menos frecuentes que los anteriormente comentados, y podrían limitar su uso en estos pacientes ${ }^{37}$.

Los cannabinoides podrían constituir una alternativa terapéutica, como tratamiento adyuvante, en el 10$30 \%$ de pacientes que presentan vómitos agudos y en el $50 \%$ que padecen náuseas a pesar del tratamiento con inhibidores de la serotonina ${ }^{38}$. También podrían ser útiles en determinados grupos de pacientes oncológicos que reciben quimioterapia moderadamente emetógena y tienen factores de riesgo que les predisponen a padecer estos efectos indeseados como son las mujeres con cáncer de mama, en las que la efectividad de los inhibidores de la serotonina no ha sido superior a la de otros antieméticos ${ }^{39,40}$. También se ha sugerido que podrían ser eficaces en el tratamiento de las náuseas y vómitos anticipatorios. No obstante, hacen falta ensayos clínicos controlados que confirmen estas hipótesis.

\section{Síndrome de anorexia-caquexia}

Los resultados de algunos ensayos clínicos de corta duración sobre el tratamiento del síndrome de anorexia-caquexia en pacientes con sida ${ }^{41,42,43}$ o con ciertos cánceres en fase terminal ${ }^{44}$, sugieren que el dronabinol es superior a placebo pero no al megestrol para aumentar el apetito y mantener el peso. La combinación de megestrol y dronabinol no parece tener efecto terapéutico aditivo. El principal inconveniente del megestrol son los episodios trombóticos y la impotencia. Se ha sugerido que el efecto immunosupressor de los cannabinoides podría limitar el uso en estos pacientes pero esta hipótesis no se ha confirmado en la practica clínica ${ }^{45,46}$. En tres estudios en pacientes con sida el uso de cannabis no aceleró la progresión de la enfermedad ${ }^{47,48,49}$. Actualmente hay en curso un ensayo en fase III para comparar la eficacia de un extracto de cannabis con la de THC ambos administrados por vía oral en el tratamiento del síndrome de anorexia-caquexia en 40 pacientes con cáncer terminal. También se evaluarán otras variables como el efecto antinauseoso y el analgésico ${ }^{50}$. 


\section{Dolor}

La eficacia analgésica de los cannabinoides ha sido evaluada en el tratamiento del dolor oncológico, neuropático y postoperatorio ${ }^{51}$. Actualmente hay diversos ensayos clínicos en curso en esta indicación ${ }^{52,53,54}$.

Una revisión de cinco ensayos clínicos en un total de 118 pacientes, sugiere que la administración de THC (10-20 mg por vía oral) tiene una eficacia analgésica superior a placebo y similar a codeína $(60-120 \mathrm{mg}$ ) en el tratamiento del dolor oncológico, aunque el THC a dosis altas $(20 \mathrm{mg}$ ) se acompaña de efectos neropsiquiátricos limitantes de la dosis ${ }^{51}$.

El uso de cannabinoides en el tratamiento del dolor neuropático se basa en los resultados de una serie de casos con nabilona y de cuatro pequeños ensayos clínicos en los cuales se ha evaluado la eficacia de diferentes cannabinoides administrados por vía oral o sublingual en pacientes con dolor secundario a esclerosis múltiple, lesión del plexo braquial, ciática por cirugía espinal, neuralgia del trigémino y algias faciales, entre otros tipos de dolor neuropático, resistente a los tratamientos analgésicos habituales $55,51,56,57,58$.

En una serie de 60 pacientes con diferentes tipos de dolor neuropático (secundario a EM, dolor neuropático central, neuropatía periférica, dolor oncológico y lumbalgia) resistente a otros tratamientos analgésicos, la nabilona $(0,25$ a $3 \mathrm{mg}$ al día) mostró algún efecto beneficioso en un 30\% de los mismos. Un 25\% no toleraron el tratamiento. Los efectos indeseados más frecuentes fueron somnolencia y disforia. Algunos pacientes refirieron a su vez mejoría de la calidad del sueño, los espasmos musculares, los síntomas urinarios, la constipación y la ansiedad. La mayoría de pacientes con experiencia de uso previo de cannabis lo prefirieron a la nabilona ${ }^{56}$.

Las pruebas clínicas disponibles sobre el tratamiento del dolor agudo se basan en el resultado de dos ensayos clínicos en fase II de búsqueda de dosis, en el cual el levonantradol (un cannabinoide sintético administrable por vía intramuscular) mostró una eficacia analgésica superior a placebo en pacientes con traumatismo y dolor postoperatorio ${ }^{51}$ y un tercer estudio en el cual una dosis única de $5 \mathrm{mg}$ de THC administrado por vía oral a les 48 horas de una intervención quirúrgica no se mostró eficaz ${ }^{59}$. Por otro lado, actualmente está en curso en el Reino Unido el ensayo clínico CANPOP (Clinical Trial as Proof of Principle of Analgesic Effectivenes of Cannabinoids on Postoperative Pain) financiado por el Medical Research Council (MRC). Su objetivo es comparar la eficacia analgésica de $10 \mathrm{mg}$ de THC, un extracto de cannabis, y paracetamol en dosis única, administrados por vía oral una hora antes de la intervención, en 400 pacientes con dolor postoperatorio (amigdalectomizados y pacientes sometidos a cirugía abdominal). La duración prevista del estudio es de un año. Se evaluarán variables de intensidad de dolor, necesidad de opioides y efectos indeseados ${ }^{52}$.

Sobre la base de los resultados de investigación básica ${ }^{60,61}$ y clínica (casos anecdóticos, series de casos y ensayos clínicos) y teniendo en cuenta las limitaciones de las alternativas terapéuticas existentes, se ha propuesto que el dolor crónico sobre todo oncológico y neuropático, serían tipos de dolor susceptibles de tratamiento con cannabinoides ${ }^{52}$. No obstante, es necesario esperar los resultados de los ensayos clínicos en curso para definir el posible lugar en terapéutica de estos compuestos como analgésicos.

\section{Espasticidad muscular}

Las pruebas clínicas disponibles sobre la eficacia de los cannabinoides en el tratamiento sintomático de la espasticidad en pacientes con EM se habían basado hasta hace poco en descripciones anecdóticas de pacientes ${ }^{29,30}$ y en el resultado de algunos ensayos clínicos con ciertas limitaciones metodológicas ${ }^{62,63,64,65}$. Recientemente se ha publicado el estudio CAMS (Cannabinoids in Multiple Sclerosis Trial) ${ }^{66}$ un ensayo clínico comparado con placebo, financiado por el Medical Research Council (MRC) del Reino Unido. La administración de THC y un extracto de cannabis, ambos administrados por vía oral, no mostraron eficacia antiespástica objetiva según la escala de Ashworth, aunque mejoró la sensación subjetiva de rigidez, dolor y la calidad del sueño. Únicamente el grupo que recibió THC solo mejoró también la movilidad según el test de caminar diez metros. No se apreciaron diferencias en cuanto al temblor, la irritabilidad, la depresión y la astenia.

Los resultados del estudio CAMS coinciden con los de otros ensayos clínicos piloto previos (publicados en forma de resumen) que sugieren que los extractos de cannabis administrados tanto por vía oral como por vía sublingual tendrían un efecto beneficioso subjetivo (según los pacientes) en el tratamiento sintomático de los espasmos musculares ${ }^{67}$, el dolor ${ }^{68}$ y la mejoría de la calidad del sueño ${ }^{69}$.

Otros ensayos clínicos han evaluado la eficacia de los cannabinoides en el tratamiento de la postura, el temblor y los síntomas urinarios de pacientes con EM. Se trata de ensayos comparados con placebo $(2$ con cannabis fumado y uno con extracto de cannabis por vía sublingual) con un numero muy limitado de pacientes (entre 8 y 20) que sugirieron una mejoría en la sensación subjetiva de temblor, hiperrreactividad de la vejiga urinaria (medida por cistometría) o una sensación general de mejoría, si bien en uno de ellos el equilibrio muscular y la postura empeoraron, según la evaluación de los investigadores ${ }^{70,71,72}$.

La compañía británica GW Pharmaceuticals está pendiente de publicar los resultados definitivos de un 
ensayo clínico en fase III en el que se ha evaluado la eficacia de sus extractos de administración sublingual para el tratamiento de la espasticidad y otros síntomas de la EM. Según la compañía los resultados preliminares de estos estudios son positivos y tiene previsto solicitar a las autoridades sanitarias británicas la aprobación de este producto para esta indicación sobre la base de los mismos ${ }^{73}$.

Los datos preclínicos y los resultados de los ensayos clínicos publicados sugieren un papel de los cannabinoides en el tratamiento de la espasticidad y otros síntomas de la EM en pacientes resistentes a otros tratamientos para los que no se dispone todavía un tratamiento totalmente eficaz ${ }^{66,74}$. Hacen falta nuevos ensayos clínicos que confirmen su lugar en terapéutica en esta indicación.

\section{Otras Indicaciones}

Sobre el uso de cannabinoides en el tratamiento de las alteraciones del movimiento, la mayoría de las publicaciones describen pequeñas series de pacientes. La eficacia de los cannabinoides en el tratamiento de la epilepsia y la corea de Huntington no está clara ${ }^{75,76}$. En un ensayo clínico en un total de 24 pacientes con síndrome de Gilles de la Tourette la administración de THC por vía oral durante seis semanas mostró una eficacia superior a placebo en el control de los tics ${ }^{77}$. Se han descrito cinco pacientes con enfermedad de Parkinson que presentaron una mejoría subjetiva del temblor después de fumar marihuana, pero esta mejoría no fue objetivada por los evaluadores ${ }^{78}$. En un estudio en siete pacientes con Parkinson, la nabilona mejoró las discinesias inducidas por levodopa ${ }^{79}$. Dos ensayos clínicos piloto sugieren que el dronabinol 2,5 mg a 5 mg dos veces al día puede aumentar el hambre, mantener el peso y disminuir la agitación en pacientes con enfermedad de Alzheimer ${ }^{80,81}$.

El THC disminuye la presión intraocular, de manera proporcional a la dosis ${ }^{82}$. Sin embargo, este efecto dura poco, porque el THC desaparece rápidamente del humor vítreo, y persiste sólo mientras el fármaco está presente. Recientemente, un cannabinoide sintético por vía tópica se ha mostrado eficaz para reducir la presión intraocular en pacientes con glaucoma que no responden a otros tratamientos ${ }^{83}$. El THC administrado en forma de aerosol ha mostrado un efecto broncodilatador similar al del salbutamol. No obstante el THC administrado por está vía produjo irritación de las vías respiratorias ${ }^{84}$.

Algunos cannabinoides se han evaluado como ansiolíticos con resultados discrepantes ${ }^{85,86,87}$. Además de las investigaciones en curso reseñadas hasta ahora, los cannabinoides se están evaluando como neuroprotectores $^{31}$ y agentes antitumorales ${ }^{32}$.
Actualmente se está realizando una rica investigación básica en modelos de enfermedades degenerativas neurológicas (esclerosis múltiple, enfermedad de Parkinson, corea de Huntington y enfermedad de Alzheimer), neuroprotección, dolor, tratamiento de la dependencia de opioides, glaucoma, cáncer (sobre todo tumores neurológicos) y regulación de la presión arterial en situaciones patológicas. Estas investigaciones permitirán conocer mejor los mecanismos básicos y desarrollar alternativas farmacológicas con efectos más específicos ${ }^{19}$.

\section{EFECTOS ADVERSOS}

Los efectos adversos del cannabis dependen sobre todo de la vía de administración y la duración de la exposición. Su toxicidad aguda es baja, dado el amplio margen terapéutico ${ }^{20}$. Los efectos euforizante, ansiolítico y a veces sedante del cannabis pueden influir en el potencial terapéutico, ya que en algunas circunstancias pueden ser percibidos como indeseados, y en otros como beneficiosos ${ }^{37}$. Sin embargo, como produce taquicardia y a veces alteración de la sensación de frío y calor, algunos pacientes pueden angustiarse 0 incluso sufrir una reacción de pánico. Los resultados de los ensayos clínicos publicados indican que un $23 \%$ de los pacientes tratados se retiraron debido a efectos adversos como mareo, flotación, depresión, distorsión de la realidad y disforia ${ }^{88}$. No se han descrito signos de dependencia en pacientes que lo usan con finalidad terapéutica. Es preciso aclarar si el THC puede participar en interacciones con otros fármacos. Por otro lado hacen falta ensayos clínicos que evalúen indeseados de los efectos de los cannabinoides a largo plazo.

\section{ESPECIALIDADES FARMACÉUTICAS}

La nabilona (Nabilone ${ }^{\circledR}$ ) está comercializada en algunos países y disponible en España, en cápsulas de $1 \mathrm{mg}$, como medicación extranjera a través de los servicios de farmacia de los hospitales. Está autorizada para la prevención de las náuseas y vómitos secundarios a quimioterapia que no han respondido a los tratamientos antieméticos de referencia (hay que justificarlo en la solicitud). Se recomiendan dosis de 2-4 mg al día, pero se pueden dar hasta $6 \mathrm{mg}$ al día, repartidos en tres tomas.

El dronabinol (Marino ${ }^{\circledR}$, Elevat $^{\circledR}$ ) está comercializado en diversos países para la prevención de náuseas y vómitos secundarios a quimioterapia que no responden a los antieméticos habituales, y para el tratamiento de la anorexia en pacientes con sida. La dosis recomendada como antiemético es de $5 \mathrm{mg} / \mathrm{m}^{2}$ al día inicialmente (en 4-5 tomas), con incrementos de 
2,5 mg, hasta un máximo de $15 \mathrm{mg} / \mathrm{m}^{2}$ al día. Para el tratamiento de la anorexia en el sida, se recomienda empezar con $5 \mathrm{mg}$ al día (en 2 tomas) que se pueden aumentar hasta un máximo de 20 mg al día.

\section{CONCLUSIONES}

El cannabis, su principal principio activo (el THC) y algunos de sus derivados han sido objeto de una amplia investigación básica, que ha permitido caracterizar el sistema endocannabinoide y su participación en la regulación de diversas funciones fisiológicas básicas. Con finalidad terapéutica, se han usado la hierba entera (fumada, ingerida o por otras vías) y sus extractos, THC natural y algún derivado sintético como el dronabinol y la nabilona. Su eficacia está evaluada sobre todo en la profilaxis y tratamiento de las náuseas y vómitos por quimioterapia antineoplásica (nabilona, dronabinol) y en el tratamiento del síndrome de anorexia-caquexia de la fase terminal de sida y de algunos cánceres (dronabinol). Los resultados de ensayos clínicos recientes sugieren que el THC y los extractos de cannabis que contienen sobretodo THC y CBD pueden ser eficaces en el tratamiento del dolor neuropático y la espasticidad y otros síntomas asociados a la esclerosis múltiple. No obstante, hacen falta nuevos ensayos clínicos que confirmen su lugar en terapéutica en las distintas indicaciones de interés.

\section{AGRADECIMIENTOS}

Este trabajo se ha realizado con el apoyo del Departament de Salut de la Generalitat de Catalunya.

\section{REFERENCIAS}

1. Duran M, Laporte JR, Capellà D. Novedades sobre las potencialidades terapéuticas del Cannabis y el sistema cannabinoide. Med Clin (Barc) 2004; 122; 390-8.

2. Anónimo. GW Pharma looks for cannabis partner. Scrip 2003;2818:8.

3. Institute For Clinical Research [formato electrónico] 2003; 1 (1): Disponible en:http://www.ikf-berlin.de [consultado 20-5-04]

4. Fundació Institut Català de Farmacología. Duran M, Laporte JR, Capellà D. Ús terapèutic del cannabis i els seus derivats 2003. Barcelona: Departament de Sanitat i Seguretat Social de la Generalitat de Catalunya, 2003.

5. Duran M, Capellà D. Novetats sobre l'ús terapèutic dels cannabinoides. BIT 2003; 15: 39-42.

6. Ozyurt E, Graham DI, Woodruff GN, McCulloch J. Protective effect of the glutamate antagonist, MK-801 in focal cerebral ischemia in the cat. J Cereb Blood Flow Metab 1988;8:138-43.

7. Costa B, Conti S, Giagnomi G, Colleoni M. The nonpsychoactive cannabis constituent cannabidiol as anti-inflammatory drug in animal model in acute inflammation. Program and abstracts of the $12^{\text {th }}$ Annual Symposium on the Cannabinoids [formato electrónico] 2002; 165 (44) Disponible en: http://www. cannabinoidsociety.org/ [consultado 06-05-04]:

8. Malfait AM, Gallily R, Sumariwalla PF, Malik AS, Andrakos E, Mechoulam, et al. The nonpsychoactive cannabis constituent cannabidiol is an oral anti-arthritic therapeutic in murine collagen-induced arthritis. Proc Natl Acad Sci USA 2000;97:9561-6.

9. Massi P, Vaccani A, Sacerdote P, Parolano D. Effects of cannabidiol, a non psychoactive constituent of marijuana, on immune system. Program and abstracts of the $12^{\text {th }}$ Annual Symposium on the Cannabinoids [formato electrónico] 2002; 165 (43). Disponible en: http://www. cannabinoidsociety.org/ [consultado 06-05-03].

10. Parker AL, Mechoulam R, Schlievert C. Cannabidiol, a non-psychoactive component of cannabis and its synthetic dimethylheptyl homolog suppress nausea in an experimental model with rats. Neuroreport 2002;13: 567-70.

11. Onaivi ES, Green MR, Martin BR. Pharmacological characterization of cannabinoids in the elevated plus maze. J Pharmacol Exp Ther 1990;253: 1002-9.

12. Pertwee RG. Pharmacology of $\mathrm{CB} 1$ and $\mathrm{CB} 2$ receptors. Pharmacol Ther 1997;74:129-80.

13. Mechoulam R, Ben Shabat S, Hanus L, Fride E, Vogel Z, Bayewitch M, et al. Endogenous cannabinoid ligandschemical and biological studies. J Lipid Mediat Cell Signal 1996;14:45-9.

14. Hanus L, Abu-Lafi S, Fride E, Breuer A, Vogel Z, Shalev $D E$, et al. 2-arachidonyl glyceryl ether, an endogenous agonist of the cannabinoid CB1 receptor. Proc Natl Acad Sci USA 2001;98:3662-35.

15. Porter AC, Felder CC. The endocannabinoid nervous system. Unique opportunities for therapeutic intervention. Pharmacol Ther 2001;90:45-60.

16. Berdyshev EV. Cannabinoid receptors and the regulation of immune response. Chem Phys Lipids 2000;108:169-90.

17. Kunos G, Jarai Z, Batkai S, Goparaju SK, Ishac EJ, Liu J, et al. Endocannabinoids as cardiovascular modulators. Chem Phys Lipids 2000;108;159-68.

18. Paria BC, Dey SK. Ligand-receptor signalling with endocannabinoids in preimplantation embryo development and implantation. Chem Phys Lipids 2000;108;211-20.

19. Piomelli D, Giuffrida A, Calignano A, Rodríguez de Fonseca F. The endocannabinoid system as target for therapeutic drugs. Trends Pharmacol Sci 2000;21:218-24.

20. Ashton $\mathrm{CH}$. Adverse effects of cannabis and cannabinoids. Br J Anaesth 1999;83:637-49.

21. Bovasso GB. Cannabis abuse as risk factor for depressive symptoms. Am J Psychiatry 2001;158:2033-37.

22. Patton GC, Coffey C, Carlin JB, Degenhardt L, Lynskey M, Hall W. Cannabis use and mental health in young people: cohort study. BMJ 2002; 325:1195-8. 
23. Pope HGJ, Gruber AJ, Hudson JI, Huestis MA, YurgelunTodd D. Neuropsychological performance in long-term cannabis users. Arch Gen Psychiatry 2001;58:909-15.

24. Solowij N, Stephens RS, Roffman RA, Babor T, Kadden $R$, Miller $M$, et al. Cognitive functioning of long-term heavy cannabis users seeking treatment. JAMA 2002;287:1123-31.

25. Grant I, Gonzalez R, Carey C, Natarajan L. Longterm neurocognitive consequences of marijuana: a meta-analytic study. A: National Institute on Drug Abuse Workshop on Clinical Consequences of Marijuana [formato electrónico] 2001(1)11. Disponible en http:// www.nida.gov/MeetSum/marijuanaabstracts.html. Accessibility [consultado 08-03-03].

26. Pope HG. Cannabis, cognition and residual confounding. JAMA 2002;287;1172-74.

27. Van Os J, Bak M, Hanssen M, Bijl RV, de Graaf R, Verdoux $H$. Cannabis use and psicosis: a longitudinal populationbased study. Am J Epidemiol 2002;156:319-27.

28. Grotenhermen F. Pharmacokinetics and pharmacodynamics of cannabinoids. Clin Pharmacokinet 2003;42:327-60.

29. The United Kingdom Parliament House of Lords. Science and Technology. Ninth report on therapeutic uses of cannabis [formato electrónico] 1998. Disponible en: http://www.parliament.the-stationery office.co.uk/pa/ Id199798/Idselect//dsctech/151/15101.htm [consultado 03-05-04].

30. Institute of Medicine. Marijuana and medicine [formato electrónico] 1999; 196 (196) Disponible en: http://www. bob.nap.edu/books/0309071550/html. [consultado 0311-03].

31. Knoller N, Levi L, Shoshan I, Reichenthal E, Razon N, Rappaport ZH, et al. Dexanabinol (HU-211) in the treatment of severe closed head injury: a randomised, placebo-controlled, phase II clinical trial. Crit Care Med 2002;30:548-54.

32. Guzmán M. Cannabinoids: Potential anticancer agents. Nat Rev Cancer 2003;3:745-55.

33. Musty RE, Rossi R. Effects of smoked cannabis and oral D9-tetrahydrocannabinol on nausea and emesis after cancer chemotherapy: A review of state clinical trials. J CANT formato electrónico] 2001;1:29-56. Disponible en: http//www.cannabis-med.org/science-international/ JCANT.btm. [consultado 30.12.03].

34. Vicinguerra $V$, Moore T, Brennan E. Inhalation marijuana as an antiemetic for cancer chemotherapy. NY State Med 1988;88:525-7.

35. Levitt M, Faiman C, Hawks R, Wilson A. Randomized double blind comparison of delta-9-tetrahydrocannabinol (THC) and marihuana as chemotherapy antiemetics. ASCO Abstracts 1984;3:91.

36. Chang AE, Shiling DJ, Stillman C, Goldberg NH, Seipp CA, Barofsky I, et al. Delta-9-tetrahydrocannabinol as antiemetic in cancer patients receiving high dose methotrexate. Ann Intern Med 1979;91:819-24.

37. Tramèr MR, Carroll D, Campbell FA, Reynolds DJ, Moore RA, McQuay HJ. Cannabinoids for control of chemotherapy induced nausea and vomiting, quantitative systematic review. BMJ 2001;323:16-21.
38. Morrow GR, Hickok JT, Rosenthal SN. Progress in reducing nausea and emesis. Cancer 1995;76:343-57.

39. Roscoe JA, Morrow GR, Hickok JT, Stern RM. Nausea and vomiting remain a significant clinical problem: Trends over time in controlling chemotherapy-induced nausea and vomiting in 1413 patients treated in community clinical practices. J Pain Symptom Manage 2000;20:113-21.

40. Hickok JT, Roscoe JA, Morrow GR, Stern RM, Yang B, Flynn PJ, et al. Use of 5-HT3 receptor antagonists to prevent nausea and emesis caused by chemotherapy for patients with breast carcinoma in community practice settings. Cancer 1999;86:64-71.

41. Beal JE, Olson R, Laubenstein L, Morales JP, Bellman P, Yangco $B$, et al. Dronabinol as a treatment for anorexia associated with weight loss in patients with AIDS. J Pain Symptom Manag 1995;10:89-97.

42. Struwe M, Kaempfer SH, Geiger CJ, Pavia AT, Plasse TF, Sherpard KV, et al. Effect of dronabinol on nutritional status in HIV infection. Ann Pharmacother 1993; 27:827-31.

43. Timpone JG, Wright DJ, Li N, Egorin MJ, Enama ME, Mayers J, et al. The safety and pharmacokinetics of single-agent and combination therapy with megestrol acetate and dronabinol for the treatment of HIV wasting syndrome. AIDS Res Hum Retroviruses 1997;13: 305-15.

44. Jatoi A, Windschitl HE, Loprinzi CL, Sloan JA, Dakhil SR, Mailliard JA, et al. Dronabinol versus megestrol acetate versus combination therapy for cancer-associated anorexia: a North Central Cancer Treatment Group Study. J Clin Oncol 2002;15:567-73.

45. Pacifici R, Zuccaro P, Pichini S, Roset PN, Poudevida S, Farre $\mathrm{M}$ i col.ls. Modulation of the immune system in cannabis users. JAMA 2003; 289: 1929-31.

46. Parolaro D. Presence and functional regulation of cannabinoid receptors in immune cells. Life Sci 1999;65:637-44.

47. Di Franco MJ, Shepard HW, Hunter DJ. The lack of association of marihuana and other recreational drugs with progression to AIDS in San Francisco Men's Health Study. Ann Epidemiol 1996;6:283-89.

48. Whitfield RM, Bechtel LM, Starich GH. The impact of ethanol and Marinol/marijuana usage on HIV+AIDS patients undergoing azidothymidine, azidothymidine/ dideoxycytine, or dideoxyinosine therapy. Alcohol Clin Exp Res 1997; 21: 122-27.

49. Abrams DI, Hilton JF, Leiser RJ, Shade SB, Elbeik TA, Aweeka FT et al. Short-term effects of cannabinoids in patients with HIV-1 infection: a randomized, placebocontrolled clinical trial. Ann Intern Med 2003;139:258-66.

50. Strasser F, Lueftner D, Possinger K, Ernst G, Ruhstaller T, Meissner $W$ et al. Oral cannabis extract versus delta-9-tetrahydrocannabinol for patients with cancer related anorexia: a randomized, double blind placebo controlled multicenter study. Program and abstracts of the IACM $2^{\text {nd }}$ Conference on Cannabinoids in Medicine [formato electrónico] 2003;35(20). Disponible en: http://www.acmed.org/spanish/nav/home-conference. htm [consultado26-5-04].

51. Campbell FA, Tràmer MR, Carroll D, Reynolds DJM, Moore RA, McQuay HJ. Are cannabinoids an effective 
and safe treatment option in the management of pain? A qualitative systematic review. BMJ 2001;323:13-6.

52. Baker D, Pryce G, Giovannoni G, Thompson AJ. The therapeutic potential of cannabis. Lancet Neurol 2003;2:291-8.

53. GW Pharmaceuticals. Research and devolopement [en formato electrónico]. Disponible en: www.gwpharm. com/corp_glan-phas-3.html [consultado 27-5-04].

54. Abrams DI, Jay C, Petersen K, Shade S, Vizoso H, Reda $\mathrm{H}$ et al. The effects of smoked cannabis in painful peripheral neuropathy and cancer pain refractory to opioids. Program and abstracts of the 2 nd conference on Cannabinoids in Medicine [en formato electrónico] 2003. Disponible en http// www.cannabismed.org/ Meeting/cologne2003/reader.pdf [consultado 26-5-04].

55. Karst M, Salim K, Burstein S, Conrad I, Hoy L, Schneider $U$. Analgesic effect of the synthetic cannabinoid CT-3 on chronic neuropathic pain. JAMA 2003; 290:1757-62.

56. Notcutt W, Prince M, Chapman G. Clinical experience with nabilone for chronic pain. Pharmaceut Sci 1997;3:551-5.

57. Berman J, Lee J, Cooper M, Cannon A, Sach J. Efficacy of two cannabis-based medicinal extracts for relief of central neuropathic pain from brachial plexus avulsion: results of a randomised controlled trial. Anaesthesia 2003;58:936-40.

58. Notcutt W, Price M, Miller R, Newport S, Phillips C, Simmons $S$, et al. Initial experiences with medicinal extracts of cannabis for chronic pain: results from 34 ' $n$ of 1 ' studies. Anaesthesia 2004;59:440-52.

59. Buggy DJ, Toogood L, Maric S, Sharpe P, Lambert DG, Rowbotham DJ. Lack of analgesic efficacy of oral delta9-tetrahydrocannabinol in postoperative pain. Pain 2003;106:169-72.

60. Pertwee RG. Cannabinoid receptors and pain. Prog Neurobiol 2001;63:569-611.

61. Rice ASC. Cannabinoids and pain. Curr Opin Investig Drugs $2001 ; 2: 399-414$

62. Petro DJ, Ellenberger Jr C. Treatment of human spasticity with delta 9-tetrahydrocannabinol. J Clin Pharmacol 1981;21 (8-9 Suppl):413S-416S.

63. Ungerleider JT, Andyrsiak T, Fairbanks L, Ellison GW, Myers LW. Delta-9-THC in the treatment of spasticity associated with multiple sclerosis. Adv Alcohol Subst Abuse 1987;7:39-50.

64. Martyn CN, Illis LS, Thom J. Nabilone in the treatment of multiple sclerosis. Lancet 1995;345:579.

65. Killestein J, Hoogervorst EL, Reif M, Kalkers NF, Van Loenen AC, Staats PG, et al. Safety, tolerability, and efficacy of orally administered cannabinoids in MS. Neurology 2002;58:1404-7.

66. Zajicek J, Fox P, Sanders H, Wright D, Vickery J, Nunn A et al. Cannabinoids for treatment of spasticity and other symptoms related to múltiple sclerosis (CAMS study): multicentre randomised placebo-controlled trial. Lacet 2003;362:1517-26.

67. Vaney C, Heinzel-Gutenbrunner M. Efficacy, safety and tolerability of an orally administered cannabis extract in treatment of spasticity in patients with multiple sclerosis: a randomized, double blind, placebo- controlled, crossover study. Multiple Sclerosis ECTRIMS 2003;9:S14.

68. Rog D, Young CA. Randomized controlled trial of cannabis-based medicinal extracts in the treatment of neuropatic pain due to multiple sclerosis. Multiple Sclerosis ECTRIMS 2003;9:S25.

69. Wade DT, Robson P, House H, Makela P, Aram J. A preliminary controlled study to determine whether whole-plant cannabis extracts can improve intractable neurogenic symptoms. Clin Rehabil 2003;17:21-9.

70. Greenberg HS, Werness SAS, Pugh JE, Andrus RO, Anderson DJ, Domino EF. Short-term effects of smoking marijuana on balance in patients with multiple sclerosis and normal volunteers. Clin Pharmacol Ther 1994;55:324-8.

71. Brady CM, Dasgupta P, Wieman OJ, Berkley K, Fowler CJ. Acute and chronic effects of cannabis based medicinal extract on refractory lower urinary tract dysfunction in patients with advanced multiple sclerosis. J Neurol Neurosurg Psych 2002;72:139.

72. Clifford DB. Tetrahydrocannabinol for tremor in multiple sclerosis. Ann Neurol 1983;13:669-71.

73. GW Pharmaceuticals. GW announces positive results from each of four phase three clinical trials [en formato electrónico]. Disponible en: http://www.gwpharma.com/ news_pres_05_nov_02.html [consultado 26-05-04]:

74. Shakespeare DT, Young CA, Boggild M. Anti-spasticity agents for multiple sclerosis (Cochrane Review). The Cochrane Library, Issue 4, 2000.

75. Karler R, Turkanis SA. The cannabinoids as potential antiepileptics. J Clin Pharmacol 1981; 21: 437S-48S.

76. Consroe P, Laguna J, Allender J, Snider S, Stern L, Sandyk R, et al. Controlled clinical trial of cannabidiol in Huntington's disease. Pharmacol Biochem Behav 1991;40:701-08.

77. Muller-Vahl KR, Schneider U, Prevedel H, Theloe K, Kolbe H, Daldrup T et al. Delta 9-tetrahydrocannabinol (THC) is effective in the treatment of tics in Tourette syndrome: a 6-week randomized trial. J Clin Psychiatry 2003;64:459-65.

78. Frankel JP, Huges A, Lees AJ. Marijuana for parkinsonian tremor. J Neurol Neurosurg Psychiatry 1990;53:436.

79. Sieradzan KA, Fox SH, Mill M, Dick JPR, Crossman AR, Brotchie JM. Cannabinoids reduced levodopa induced dyskinesias Parkinson's disease. A pilot study. Neurology 2001;57:2108-11.

80. Volicer L, Stelly M, Morris J, McLaughlin J, Volicer BJ. Effects of dronabinol on anorexia and disturbed behavior in patients with Alzheimer's disease. Int J Geriatr Psychiatry 1997;12:913-9.

81. Ross JS, Shua-Haim JR. Open label study of dronabinol in treatment of refractory agitacion in Alzheimer's disease: a pilot study. Program and abstracts of 34th Congress of ASCP [en línea] 2003 Novembre 14. EurekAlert [accedido el día 7 de junio de 2004]; 1 (1). URL disponible en http://www.cannabis-med.org/ studies/study.php

82. Hepler RS, Frank IR. Marihuana smoking and intraocular pressure JAMA 1971;217:1392.

83. Porcella A, Maxia C, Gessa GL, Pani L. The synthetic cannabinoid WIN55212-2 decreases the intraocular 
pressure in human glaucoma resistant to conventional therapies. Eur J Neurosci 2001;13:409-12.

84. Tashkin DP, Reiss S, Shapiro BJ, Calvarese B, Olsen JL, Lodge JW. Bronchial effects of aerosolized delta 9 tetrahydrocannabinol in healthy asthmatic subjects. Am Rev Respir Dis 1977;115:57-65.

85. Fabre LFR, McLendon D. The efficacy and safety of nabilone (a synthetic cannabinoid) in the treatment of anxiety. J Clin Pharmacol 1981;21 (Suppl 8-9):377S-82S.
86. Glass RM, Uhlenhuth EH, Hartel FW. The effects of nabilone, a synthetic cannabinoid on anxious human volunteers. Psychopharmacol Bull 1979;15:88.

87. Glass RM, Uhlenhuth EH, Hartel FW. Single dose study of nabilone in anxious volunteers. J Clin Pharmacol 1981;21S:383

88. Voth EA, Schwartz RH. Medicinal applications of delta9-tetrahydrocannabinol and marijuana. Ann Intern Med 1997;126:791-8.

\section{FE DE ERRATAS}

En el volumen 16, suplemento $n^{\circ} 1$, en el que se editaron los resúmenes de las ponencias de las XXXI JORNADAS NACIONALES DE SOCIDROGALCOHOL, los autores de la ponencia titulada MUJER Y ADICCIONES: LAS SALIDAS DE UN LABERINTO son S. Cazorla, M. Conderana y M. Moreno, apareciendo otros en su lugar por error. 